\title{
A TDM-BASED CONTROL MECHANISM WITH GROUP INTERLEAVED CONTROL CHANNEL FOR WAVELENGTH DIVISION MULTIPLEXING NETWORKS
}

\author{
Chi-Yuan Chang and Sy-Yen Kuo \\ Department of Electrical Engineering, National Taiwan University, Taipei, Taiwan \\ chiyuan@ion.ee.ntu.edu.tw, sykuo@cc.ee.ntu.edu.tw
}

\begin{abstract}
Various network topologies, such as bus, ring and star, as well as WDM (Wavelength division multiplexing) and TDMA(Time Division Multiplexing Access) are popular in MAN/LAN. However, TDMA is suitable only for a heavily loaded traffic. On the other hand, the network traffic in these applications such as multimedia, videoconferencing, etc., usually exhibits traffic locality. This type of traffic uses relatively high bandwidth on a continuous basis for a long period of time. As a result, traditional TDMA is no longer appropriate for such traffic because of inefficiency. Consequently, based on the traffic parameters such as locality and loading, an architecture named as rGDBLN (r Group Dual Bus Lightwave Network), which partitions/reconfigures the network into several control groups, is proposed. Furthermore, the interleaved control slot (ICS) equipped with cross-group section (CGS) and non-cross-group section (NCGS) for reducing collisions is also presented. The slot reuse can be easily achieved by using the ICS scheme and the slot utilization of the network is superior to the original network under the specific traffic.
\end{abstract}

\section{INTRODUCTION}

Many new applications such as high resolution image, distributed database, real-time video/audio service with high performance needs require more bandwidth/quality than ever before. These applications generally ask the data to be transmitted very fast under heavy traffic in a metropolitan area network (MAN) or local area network(LAN). Optical fiber has been widely adopted for satisfying these requirements. The wavelength division multiplexing (WDM) technique [1] is widely employed to fully utilize the huge bandwidth available on optical fibers and has contributed significantly to high speed communications. The WDM technique divides the optical bandwidth of a fiber into a number of smallercapacity channels operating at full electronic speed, say a few $\mathrm{Gb} / \mathrm{s}$. A very high throughput can be achieved by concurrently transmitting data belonging to different users on channels at different wavelengths.

- There are three basic physical network topologies for multiaccess WDM network: the bus, the star, and the ring. Other types of topologies can be easily extended with these basic topologies. A significant development in the data communication field has been the standardization of distributed queue dual-bus (DQDB) protocol as the IEEE 802.6 metropolitan area network (MAN)[2]. Several multichannel bus/ring lightwave networks [3-8] had been

\footnotetext{
\%. This research was supposed by the National Science Council, Taiwan, R. O. C., under the Grant NSC 872213-E259-007
}

reported. In the past, many researchers $[7,8]$ have taken advantage of TDMA (Time Division Multiplexing Access) technique in the WDM environment. TDMA is a direct solution to achieve fair transmission. TDMA corresponds to a fixed partitioning of the channel bandwidth among all the possible transmitters. It does not allow a node to grab more than the portion of the channel bandwidth assigned to it even if no other nodes are transmitting on the same channels. Hence, TDMA is suitable only under a heavily loaded traffic. For a lightly loaded traffic, the delay performance of the TDMA is very poor because a node must wait for the assigned slot before transmitting a newly generated packet.

Many network applications such as multimedia, videoconferencing, etc., usually exhibit traffic locality (which means the traffic between the transmitter node and the receiver node is located at the same area) $[7,8]$. This type of traffic uses relatively high bandwidth on a continuous basis for a long period of time, while the data traffic is burst, but the average bandwidth used is low. Besides, for traffic with higher priority, the network can also route the traffic as soon as possible by sacrificing the lower priority traffic. Although the network with WDM and TDMA is popular, however, traditional TDMA is no longer suitable for such traffic because of inefficiency and non multi-level priority. For the sake of simplicity, we restrict our discussion in the following on the bus topology. Of course, our proposed methodology can not only be implemented in bus architecture, but also be applied to other topologies such as ring or star. Hence, based upon traffic locality and loading (light or heavy) characteristics, an architecture named as rGDBLN (r Group Dual Bus Lightwave Network) is proposed to partition/reconfigure the network into several control groups. The rGDBLN consists of $r$ different groups of stations. All stations in each group have the traffic locality and the same control channel. The control channel named as the interleaved control slot (ICS) is arranged by overlapping half cycle. Each control slot is equipped with two sections: cross-group section (CGS) and non-cross-group section (NCGS). By adequate partitioning/reconfiguring network and using ICS as control protocol, the slot reuse can be easily achieved and the slot utilization of the network is superior to the original network under the specific traffic.

This paper is organized as follows. In section 2 we provide the network topology, introduce the interleaved control slot concept and present the assignment of wavelength. Section 3 discusses the traffic types and presents two algorithms to reconfigure the network for dynamic loading balance. Section 4 depicts a simulation model and the simulation results. In section 5 we 
conclude the paper.

\section{NETWORK TOPOLOGY}

In Figure 1, the dual bus lightwave network (DBLN) is considered and each node is equipped with some tunable transmitters (TTs) and a fixed receiver (FR). The DBLN consists of two optical buses, Bus A and Bus B, respectively. Each bus has $k+1$ wavelengths /channels $\left(\lambda_{0}, \lambda_{1}, \lambda_{2}, \cdots, \lambda_{k}\right)$ by employing the WDM technology. The DBLN consists of $\mathrm{N}$ stations which are labeled from left (upstream) to right (downstream) as 1 to $\mathrm{N}$ with respect to Bus $\mathrm{A}$. The stations near the head of Bus $A$ are referred to as the upstream stations and those near the head of Bus B downstream stations.

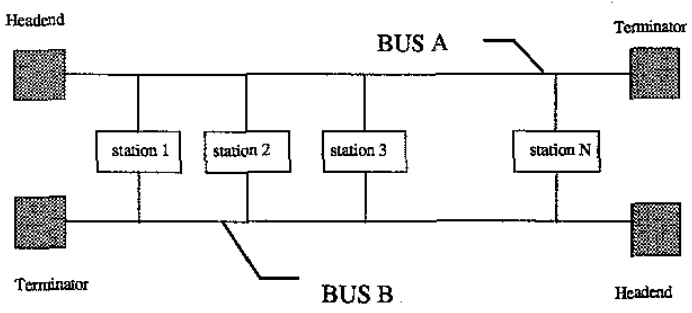

Figure 1. The architecture of a dual bus lightwave network.

The traditional approach uses a wavelength to be the control channel to decide which station could transmit data[4,5,7-8]. Hence, Each station has one fixed transmitter for receiving control channel which is TDMbased. The pseudo cycle with a length of $\mathrm{N}$ control slots is generated on the control channel, and only one control slot is allocated for each station in a cycle. On the other hand, a tunable transmitter is used for transmitting data to destination and a fixed/tunable receiver is used for receiving data form source. For the sake of simplicity, we assume that the number of wavelengths is enough for distributing the stations, that is $k>=N$. This implies that conflict case can be avoided and is temporarily not taken into consideration now. The other cases such as $k<N$ are discussed later. Figure 2 shows the channel assignment of network with $N=8, k=8$. The station is equipped with a fixed receiver tuned to $\lambda_{0}$ for access control channel, a tunable transmitter tuning wavelengths form $\lambda_{1}$ to $\lambda_{8}$ and a fixed receiver. When a station $i$ want to transmit data to station $j$, there are several steps to setup before transmitting. First, the tunable $T x$ need to tune its wavelength to $\lambda_{j}$ and then wait for transmitting data until the $i t h$ control slot is accessed by the control channel. Station $i$ can only transmit in the interval time at the $i t h$ control slot. If station $i$ needs to transmit data again, it must wait until the ith control slot of next control cycle. It is clear that each station must wait for $n$ control slots to transmit data again. If station $i$ has a great deal of data to transmit to station $j$, the TDM-based control channel is very inefficient. As a result, a new architecture called rGDBLN ( $r$ Group DBLN) is proposed to resolve this drawback to improve performance.

\begin{tabular}{|c|c|c|c|c|c|c|c|c|}
\hline Station & 1 & 2 & 3 & 4 & 5 & 6 & 7 & 8 \\
\hline control ch. & $\lambda_{0}$ & $\lambda_{0}$ & $\lambda_{0}$ & $\lambda_{0}$ & $\lambda_{0}$ & $\lambda_{0}$ & $\lambda_{0}$ & $\lambda_{0}$ \\
\hline $\mathrm{Tx}$ & $\lambda_{1}, \cdots$ & $\lambda_{1}, \cdots$ & $\lambda_{1}, \cdots$ & $\lambda_{1}, \cdots$ & $\lambda_{1}, \cdots$ & $\lambda_{1}, \cdots$ & $\lambda_{1}, \cdots$ & $\lambda_{1}, \cdots$ \\
& $\lambda_{8}$ & $\lambda_{8}$ & $\lambda_{8}$ & $\lambda_{8}$ & $\lambda_{8}$ & $\lambda_{8}$ & $\lambda_{8}$ & $\lambda_{8}$ \\
\hline $\mathrm{Rx}$ & $\lambda_{1}$ & $\lambda_{2}$ & $\lambda_{3}$ & $\lambda_{4}$ & $\lambda_{5}$ & $\lambda_{6}$ & $\lambda_{7}$ & $\lambda_{8}$ \\
\hline
\end{tabular}

Figure 2. The channel assignment of nodes in a network.

The rGDBLN is a network which consists of $r$ groups of stations. All stations in a group are equipped with the same wavelength for control channel. Each station has one tunable transmitter tuned to a proper wavelength for transmitting data, one fixed receiver for receiving data, one tunable receiver for receiving proper control signals, and one tunable receiver for detecting the collision. The difference between the DBLN and the rGDBLN is that the DBLN is equipped with a fixed receiver for accessing the control slot, while the rGDBLN has a tunable receiver for accessing the control slot in each group. Figure 3 shows the channel assignment of stations in a rGDBLN, $r=2$, in which stations $1,3,4,7$ and stations $2,5,6,8$ are grouped together using different control wavelength $\lambda_{0}$ and $\lambda_{0}$, respectively. Obviously, Figure 2 is a special case of rGDBLN with $r=1$. Of course, the control channels of different groups can adopt the subcarrier technique $[9,10]$ if the same control wavelength $\lambda_{0}$ is used. For instance, the wavelength $\lambda_{0}$ with subcarrier $f_{1}$ and $f_{2}$ is used for stations $1,3,4,7$ and $2,5,6,8$, respectively. Since there are only $N / 2=4$ stations in a group, the average waiting time of a station for transmitting data in the rGDBLN is less than that in the DBLN as shown in Figure 2. If we assume that the traffic in the same group exhibits locality, the slot utilization is almost twice than that of the original DBLN. However, this is an upper bound of slot utilization for 2 GDBLN if full traffic locality exists in each group.

\begin{tabular}{|c|l|l|l|l|l|l|l|l|}
\hline Station & 1 & 2 & 3 & 4 & 5 & 6 & 7 & 8 \\
\hline Control ch. & $\lambda_{0}$ & $\lambda_{0}$ & $\lambda_{0}$ & $\lambda_{0}$ & $\lambda_{0}$ & $\lambda_{0}$ & $\lambda_{0}$ & $\lambda_{0}$ \\
\hline $\mathrm{Tx}$ & $\begin{array}{l}\lambda_{1}, \cdots, \\
\lambda_{8}\end{array}$ & $\begin{array}{l}\lambda_{1}, \cdots, \\
\lambda_{8}\end{array}$ & $\begin{array}{l}\lambda_{1}, \cdots, \\
\lambda_{8}\end{array}$ & $\begin{array}{l}\lambda_{1}, \cdots, \\
\lambda_{8}\end{array}$ & $\begin{array}{l}\lambda_{1}, \cdots, \\
\lambda_{8}\end{array}$ & $\begin{array}{l}\lambda_{1}, \cdots, \\
\lambda_{8}\end{array}$ & $\begin{array}{l}\lambda_{1}, \cdots, \\
\lambda_{8}\end{array}$ & $\begin{array}{l}\lambda_{1}, \cdots \\
\lambda_{8}\end{array}$ \\
\hline $\mathrm{Rx}$ & $\lambda_{1}$ & $\lambda_{2}$ & $\lambda_{3}$ & $\lambda_{4}$ & $\lambda_{5}$ & $\lambda_{6}$ & $\lambda_{7}$ & $\lambda_{8}$ \\
\hline
\end{tabular}

Figure 3 . The channel assignment of node in a rGDBLN, $\mathrm{r}=2$.

\subsection{Interleaved Control Slot Mechanism}

In this section, the control slot arrangement is proposed in order to get good slot utilization in networks. There are many solutions to resolve collision. For example, the subcarrier technique can be used to avoid the collision. That is, the receivers of all stations are equipped with channel inspection [9]. Apart from channel inspection, we propose a slot-interleaved mechanism as shown in 
Figure 4 to prevent the collision. Figure 4 shows the slotinterleaved control slot for $r=2$, where station $1,3,4,7$ are named as group 1 and stations 2,5,6,8 are named as group 2. In Figure 4 each control slot consists of 2 parts: cross-group section (CGS) and non-cross-group section (NCGS), where the CGS allows a station to transmit data to destination in different groups and the NCGS can not transmit data across different groups. For example, for stations in group 1 , if station 3 wants to transmit data to stations in group 1 such as station 1,4 ,or 7 , it can transmit data during full control slot containing the CGS and the NCGS. However, if station 3 wants to transmit data to stations in group 2 such as station $2,5,6$ or 8 , it can only transmit data in the CGS, that is a half of control slot. Owing to the traffic locality of the stations in the same group, the cross-group traffic is less than the traffic within the same group. As a result, the probability of collision will be very small and collision should be at most one half of each control slot. In order to detect the collision, a special unit named as the collision manager will be described here. The collision manager which is a tunable receiver in each station monitors the transmitter wavelength to decide whether to transmit data or not. If the collision manager detects the wavelength that has been used by other stations, then wait and try again in the next control cycle.

\begin{tabular}{|c|c|c|c|c|c|c|c|}
\hline Group 1 & 1 & & 1 & & 3 & & $\ldots$ \\
\hline Group 2 & & & & 2 & & 5 & \\
\hline
\end{tabular}

Figure 4. An example of slot-interleaved control slot.

\subsection{Assignment of Receiver Wavelength}

In this section, the assignment of wavelength is described. We consider a network with $N$ stations equipped with $r$ control channels and $k$ data channels. For the sake of easy analysis, we only take the data channel and the number of stations into consideration. In general, there are three different cases described as follows.

1. One channel per destination (node $N=$ channel $k$ ) :

In this case, each node has the sole wavelength for receiving data. For instance, in Figure 2 the receiving wavelength of node $i$ is $\lambda_{i}$. When a packet is ready for transmission from node $i$ to node $j$, the tunable transmitter of node $i$ is tuned to $\lambda_{j}$ for transmitting data until slot arrives.

\section{Less channels per destination (node $N>$ channel $k$ ):}

Since the number of logical channels $k$ is less than the number of nodes in a network, wavelength sharing is used. First, for simplicity, we restrict to network topology in which the number of channels $k$ is a divisor of the number of nodes $N$. Therefore, the number of destination nodes in each channel is equal, i.e. $N / k=r$, the $\mathrm{r}$ destinations sharing the $i t h$ channel, $0 \leq i \leq k$. The receiving wavelength of node $i$ is $\lambda_{\left.i\right|_{M}}$ (The notation $1 . l_{M}$ indicates the modulo operator). When a packet is ready for transmission from node $i$ to node $j$, the tunable transmitter of node $i$ is tuned to $\lambda_{|j|_{M}}$ for transmitting data until its next slot. Figure 5 shows the channel assignment with $k=4, N=8$ and $r=2$. Due to wavelength sharing, the collision will occur. Some mechanisms such as subcarrier $[9,10]$, TDMA technique $[5,6,7-8]$ and interleaved control slot described here could be adopted to solve the collision problem.

3. More channels per destination (node $N<$ channel $k$ ):

As already mentioned, a node is equipped with one fixed wavelength receiver for receiving data. Hence, this case in which the number of node is less than number of channels has the sole wavelength for receiving data.

\begin{tabular}{|c|c|c|c|c|c|c|c|c|}
\hline Station & 1 & 2 & 3 & 4 & 5 & 6 & 7 & 8 \\
\hline Control ch. & $\lambda_{0}$ & $\lambda_{0}$ & $\lambda_{0}$ & $\lambda_{0}$ & $\lambda_{0}$ & $\lambda_{0}^{\prime}$ & $\lambda_{0}$ & $\lambda_{0}^{\prime}$ \\
\hline $\mathrm{Tx}$ & $\begin{array}{l}\lambda_{1}, \cdots, \\
\lambda_{8}\end{array}$ & $\begin{array}{l}\lambda_{1}, \cdots \\
\lambda_{8}\end{array}$ & $\begin{array}{l}\lambda_{1}, \cdots \\
\lambda_{8}\end{array}$ & $\begin{array}{l}\lambda_{1}, \cdots \\
\lambda_{8}\end{array}$ & $\begin{array}{l}\lambda_{1}, \cdots, \\
\lambda_{8}\end{array}$ & $\begin{array}{l}\lambda_{1}, \cdots, \\
\lambda_{8}\end{array}$ & $\begin{array}{l}\lambda_{1}, \cdots \\
\lambda_{8}\end{array}$ & $\begin{array}{l}\lambda_{1}, \cdots \\
\lambda_{8}\end{array}$ \\
\hline $\mathrm{Rx}$ & $\lambda_{1}$ & $\lambda_{2}$ & $\lambda_{3}$ & $\lambda_{4}$ & $\lambda_{1}$ & $\lambda_{2}$ & $\lambda_{3}$ & $\lambda_{4}$ \\
\hline
\end{tabular}

Figure 5. The channel assignment of node in network with $N=8, k=4$.

\section{TRAFFIC AND LOAD BALANCE}

In this section the traffic representation, the network manager, and the network loading balance are discussed. Some types of traffic may be justified by traffic matrix. Based on these information, the network manager issues the reconfiguration command to stations to change the network configuration based on a dynamic loading balance.

\subsection{Traffic Representation and Network Manager}

We represent the bandwidth requirements of sourcedestination pairs by a traffic demand matrix $T=\left[t_{i j}\right][10]$. The values of $t_{i j}$ is a measure of the traffic originating at node $i$ and terminating at node $j$. However, the traffic originating and terminating at the same node is illegal in this paper and therefore, the diagonal element of $T$ is zero, i.e. $t_{i j}=0$ for $i=j$. For example, a traffic demand matrix $T$ is shown in Figure 6, in which the values of $t_{23}$ means 4 demands of the traffic originating at node 2 and terminating at node 3 .

The upper triangular matrix of $T$ denoted as $U$ is the traffic demand matrix of Bus $A$ and the lower triangular matrix of $T$ denoted as $L$ is that of Bus $B$. Hence, the total bandwidth requirement $B_{j}$ of receiver $j$ in Bus $A$ is the sum of the elements of the $j t h$ column of $U$ and total transmission requirement $T_{i}$ of node $i$ in Bus $A$ is the sum 
of the elements of the $i t h$ row of $U$. By the same way, the total bandwidth requirement $B_{j}$ of receiver $j$ in Bus $B$ is the sum of the elements of the $j t h$ column of $L$ and the total transmission requirement $T_{i}$ of node $i$ in Bus $B$ is the sum of the elements of the $i t h$ row of $L$. As a results, the following equations are derived :

$$
\begin{aligned}
& B_{j}=\sum_{i=1}^{j-1} t_{i j} \quad j=1, \cdots, N \text { for } \text { Bus } A \\
& B_{j}=\sum_{i=j+1}^{N} t_{i j} \quad j=1, \cdots, N \text { for } B \text { us } B \\
& T_{i}=\sum_{j=1}^{j-1} t_{i j} \quad i=1, \cdots, N \text { for } \text { Bus } A \\
& T_{i}=\sum_{j=i+1}^{N} t_{i j} \quad i=1, \cdots, N \quad \text { for } \quad \text { Bus } B
\end{aligned}
$$

\begin{tabular}{|c|c|c|c|c|c|}
\hline & 1 & 2 & 3 & 4 & 5 \\
\hline 1 & & 13 & 1 & 2 & 5 \\
\hline 2 & 2 & & 4 & 22 & 3 \\
\hline 3 & 13 & 1 & & 0 & 2 \\
\hline 4 & 0 & 22 & 7 & & 1 \\
\hline 5 & 11 & 11 & 6 & 11 & \\
\hline
\end{tabular}

Figure 6. A traffic demand matrix $T$.

In general, the traffic matrix represents the bandwidth requirements of source-destination pairs. The traffic status in lightly loaded condition or heavily loaded condition can be obtained by $B_{j}$ and $T_{i}$ from the traffic matrix. Moreover, the traffic parameters such as locality or loading could be justified by the traffic matrix. In the following, two types of traffic are considered.

1. general traffic (random):

If the type of traffic is random, the values of $t_{i j}$ should be randomly distributed.

2. special traffic:

The information of special traffic such as multicasting traffic, video conferencing or voice traffic can be observed from the traffic matrix. Because these types of traffic use relatively high bandwidth on a continuous basis for a long period of time and usually exhibits traffic locality, the values of these types in traffic matrix are symmetrical or their value are larger than peripheral elements. For example, in figure 6 the values of $t_{24}$ and $t_{42}$ may be video conferencing or voice traffic. On the other hand, the stations $1,2,4$ and 5 in bus $B$ may exhibit multicasting traffic because of equal values ( $t_{51}=t_{52}=t_{54}$ ).

There are many questions to be accounted in the networks. For example, how to get the traffic load of network, when to switch (that is the nodes change to different control channels) and how to change it. It can be used by a network manager [4] to evaluate the load conditions of the network in order to decide when to activate of reconfiguration network. In this way, we can know the load distribution along the buses and decide to switching reconfiguration the network. The network manager will issue the switching command including switching and grouping information after a fixed period of time. When the network manager issues the switching command to all nodes via control channel. Then all stations stop transmitting data, tune control channel according to grouping information on control channel, and then re-transmit data. This switching command information can be placed in the reserved bit of control field. On the other hand, the network manager can also be equipped with programmable group capability. For the stations with special traffics such as multicasting traffic, video conferencing or voice traffic, the network manager can be able to assign some dedicated group for special application to derive better performance.

Reconfigures the network topology based on dynamic loading balance is very important issue. It includes how to assign the nodes to different control channels in order to achieve the highest throughout and the bandwidth balance, i.e. traffic is spread across the various channel as evenly as possible. In the following, the dynamic loading balance is discussed.

\subsection{Dynamic Loading Balance}

In this section, a network reconfiguration mechanism based on a dynamic loading balance is taken into consideration. An algorithm named as CGA (Control Group Algorithm) for implementing dynamic loading balance are proposed. The CGA cares about the loading balance in each control slot for single bus $A$ or bus $B$ and the algorithm is listed as follows.

CGA algorithm : loading balance in control slot input : the traffic matrix $T$ output : $r$ group control sets, $r=2$ begin l

1. search for all remaining $T_{i j}$ in $U / L$

2. Select the biggest value of $T_{i j}$ in $U / L$ and remove ith row and jth column in $U / L$, then insert station $i$ and $j$ to group 1

3. Select the biggest value of $T_{i j}$ in $U / L$ and remove ith row and $j$ th column in $U / L$, then insert station $i$ and $j$ to group 2 4. search for all remaining $T_{i j}$ in $U / L$, repeat step 2 to 3
until all stations are selected.

$l$

end

\section{PERFORMANCE ANALYSIS}

In the following, we analyze the network performance by comparing the relationship between the slot utilization and the number of groups. Each bus has $k$ wavelengths /channels $\left(\lambda_{1}, \lambda_{2}, \cdots, \lambda_{k}\right)$ by employing the WDM technology, where channels $\lambda_{1}, \lambda_{2}, \cdots, \lambda_{I}$ are dedicated for control and others $\lambda_{r+1}, \lambda_{r+2}, \cdots, \lambda_{k}$ are for data. 


\subsection{An Upper Bound on Slot Utilization}

The slot utilization in each group is dominated by the collision probability. If the source and the destination stations of the traffic are located in the same group, i.e. the traffic is fully local, the collision will not occur and the slot utilization is improved. As a result, the slot utilization is proportional to the traffic locality and the number of groups. In order to derive the upper bound of slot utilization, the collision is assumed to be nonexistent. In addition, we assume that the slot rate is $k$ slots/sec on each control channel and the total number of stations is $N$. Hence, the $N$ stations need $N k$ slots $/ \mathrm{sec}$ for servicing. On the other hand, if the network consists of $r$ groups (control channels) where the traffic of stations in the same group is fully locally distributed, the slot rate is still $k$ slots/sec. Obviously, the $N$ stations need only $N k / r$ seconds for servicing. Figure 7 shows the upper bound of slot utilization in different groups, in which the number of stations in each group is assumed equal.

\begin{tabular}{|l|c|c|c|}
\hline Group \# & 1 & 2 & 3 \\
\hline \# of node in group & $N$ & $N / 2$ & $N / 3$ \\
\hline Upper bound of slot utilization & 1 & 2 & 3 \\
\hline
\end{tabular}

Figure 7. The upper bound of slot utilization vs. the number of nodes in different groups.

Figure 7 shows the ideal case in which there is no collision. However, this is not a real case in practical situation. Hence, the results in Figure 7 are the upper bound of slot utilization. For example, when the number of groups is 2, the number of nodes in each group becomes $n / 2$, the average waiting time of a node for transmitting data needs only $n / 2$ time slot. As a result, we can find that the upper bound of slot utilization, compared with one control channel, is $r$ times if the network is partitioned into $r$ groups.

\subsection{Simulation Models and Results}

In this section, we assume the message arrival rate of each station $i$ follows the Poisson distribution with a mean $\lambda$, and the message length follows the exponential distribution with a mean of $L$. The station load $(S L)$ for station $i$ can be defined as

$$
S L_{i}=\lambda \times L
$$

The network load (denoted as $N L$ ) can be defined as

$$
N L=\sum_{1}^{N} S L_{i} .
$$

Since the phenomena of traffic localities usually happen in real networks, the traffic source-destination distribution is derived form the following equality [3]:

$$
P_{i j}=\left\{\begin{array}{cc}
0 & j \leq i \\
\frac{(1-p)^{j-i-1} p}{1-(1-p)^{N-i+1}} & j>i
\end{array}\right.
$$

This equation represents a normalized geometric distribution where $p(0 \leq p \leq 1)$ determines the level of traffic locality. Besides, we assume that the reconfiguration time of network is regarded as a uniform distribution (i.e., reconfiguring topology is completed within specified time period). The measures of interest are the relationship among the slot utilization, the traffic locality and the network loading. The other assumptions are listed as follows:

1) $N=20$ stations in the network

2) $\mathrm{r}=\{1,2,3\}$

3) $\mathrm{NL}=\{5$ (light loading), 30 (heavy loading) $\}$

4) $\mathrm{p}=\{0.0$ (uniform distributed) $0.1,0.2,0.3,0.4$, $0.5,0.6,0.7,0.8,0.9,1.0$ (full locality))

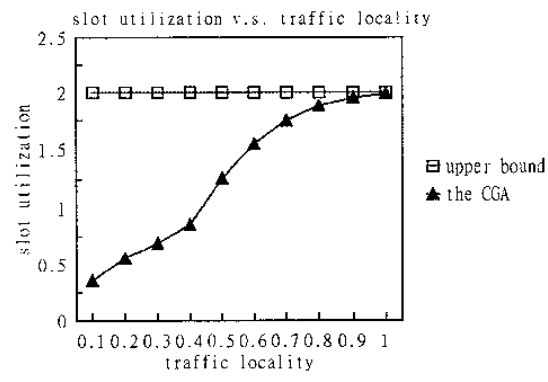

Figure 8. The slot utilization in $\operatorname{rGDBLN}$ with $r=2$.

Figure 8 shows the slot utilization in the rGDBLN network with $r=2$ and the traffic locality obtained by the CGA algorithm. The increased traffic locality could increase the slot utilization. Figure 8 illustrates that the slot utilization is large than 1 under the traffic locality $>$ 0.5 . If the traffic locality is equal to 1 ,i.e. full locality, the slot utilization almost reaches its upper bound. However, if the traffic locality is less than 0.5 , the slot utilization is below 1 due to the increasing collision probability.

In order to observe the relationship between the slot utilization and the number of groups under different traffic locality, we compare the cases of $r=1, r=2, r=3$ of rGDBLN under different network loadings in Figure 9. Figure 9a shows the slot utilization as a function of different groups under light network loading $(N L=5)$. Obviously, the traditional TDMA is a special case of rGDBLN with $r=1$, the simulation results show the slot utilization is very poor compared with $r=2,3$ for a lightly loaded traffic. We can conclude that the increased traffic locality could increase the slot utilization under light network loading shown in Figure 9a. Figure 9b shows the slot utilization as a function of different groups under heavy network loading $(\mathrm{NL}=30)$. The simulation results show that the traditional TDMA case of rGDBLN with 
$r=1$ has the best utilization. The increased number of groups has no benefit in slot utilization. On the contrary, due to the high increasing collision probability, the slot utilization is below 1 . Hence, the simulation results as shown in Figure 9 could be easily explained by the fact that the traditional TDMA technique does not allow a node to grab more than the portion of the channel bandwidth assigned to it even if no other nodes are transmitting on the same channels. However, for a lightly loaded traffic, the slot utilization of the TDMA is very poor. A group control slot can be adopted to improve the slot utilization. Therefore, we can make the suggestion that choose the TDMA if the network is heavily loaded without traffic locality, otherwise choose rGDBLN with high traffic locality.

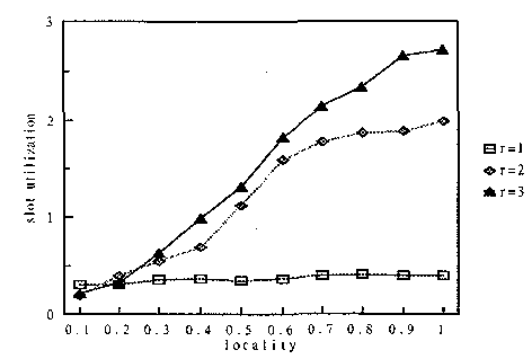

(a)

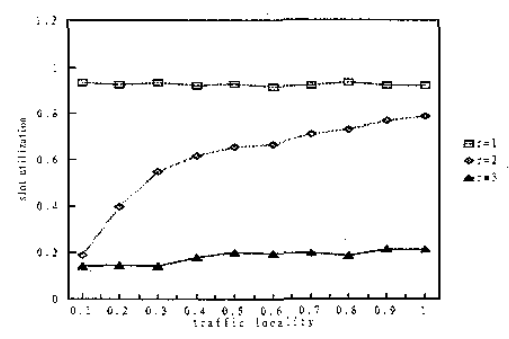

(b)

Figure 9. Slot utilization vs. number of groups with different traffic locality.

\section{CONCLUSIONS}

In this paper we have proposed an architecture named as rGDBLN. The control group has the same wavelength for control channel constructed by slot-interleaved structure. Each control slot consists of 2 parts: cross-group section (CGS) and non-cross-group section (NCGS), where the CGS allows a station to transmit data to destinations in different groups and the NCGS can not transmit data across different groups. The virtual topology of the networks, i.e. control groups, is reconfigured by the traffic locality in the networks. Owing to the traffic locality of the stations in the same group, the cross-group traffic is less than the traffic within the same group. As a result, the probability of collision will be very small and collision should be at most one half of each control slot. Besides, in order to detect the collision, a special unit named as the collision manager was proposed. If the collision manager detects the wavelength that has been used by other stations, then wait and try again in the next control cycle. We also proposed a CGA algorithm to support loading balance in dual bus for improving the network performance.

The simulation results show that the increased traffic locality could increase the slot utilization in light traffic. Because the slot utilization of the traditional TDMA is very poor in light traffic, the results suggest an alternative to choose. On the other hand, the simulation results also indicate that the traditional TDMA has the best slot utilization than rGDBLN under the heavy load. Therefore, the TDMA is a better option in heavily loaded networks under random traffic. Otherwise the rGDBLN is a good option in networks with high traffic locality.

\section{REFERENCES}

[1] C. A. Brackett, "Dense Wavelength Division Multiplexing Networks: Principles and Applications," IEEE J. Select. Areas Commu., vol. 8 , no. 6, pp. 948-964, August 1990.

[2] IEEE standards Board, IEEE standards for Local and Metropolitan Area Networks: Distributed Queue Dual Bus (DQDB) Subnetwork of a Metropolitan Area Network (MAN), IEEE std. 802.6-1990, 1990.

[3] M. A. Rodrigues, "Erasure nodes: performance improvements for the IEEE 802.6 MAN," Proc. IEEE INFOCOM'90, San Francisco, CA, pp. 636643,1990 .

[4] Wonhong Cho, Cheul Shim, and Sang-Bae Lee, "MCDODB (Multi-channel DODB) using WDM," Proc. IEEE INFOCOM'90, San Francisco, CA, pp. 2205-2209, 1995.

[5] M. A. Marsan, A. Bianco, E. Leonardi and S. Toniolo, "An Almost Optimal MAC Protocol for All-Optical WDM Multi-Rings with Tunable Transmitters and Fixed Receivers," ICC., pp. 437-442, 1997.

[6] J. C. Lu and L. Kleinrock, "A WDMA Protocol for Multichannel DQDB Networks," International Journal of Satellite Communications, vol. 9, pp. 23-35, Jan-Feb, 1991.

[7] N. F. Huang and S. T. Sheu, "An Efficient Wavelength Reusing/Migrating/Sharing Protocol for Dual Bus Lightwave Networks," IEEE J. Lightwave Tech., vol. 15, no. 1, pp. 62-75, January, 1997.

[8] N. F. Huang and S. T. Sheu, "A Wavelength Reusing/Sharing Access Protocol for Multichannel Photonic Dual Bus Networks," IEEE J. Lightwave Tech., vol. 14, no. 5, pp. 678-692, May, 1996.

[9] M. Ajmoni Marsan, A. Fumagallim E. Leonardi, F. Neri, "R-Daisy: an All-Optical Packet Network," EUROPTO European Symposium on Advanced Networks and Services, Amsterdam, Holland, March 1995.

[10] Chen-Ken Ko, and Sy-Yen Kuo, "Multiaccess Processor Interconnection Using Subcarrier and Wavelength Division Multiplexing," IEEE J. Lightwave Tech., vol. 15 , no. 2 , pp. 228-241, FEB, 1997. 